\title{
An Almost Noise-Free Power Estimation Method in Maximum Entropy Spectral Analysis
}

\author{
M.M. DAQuAH and I.H. ZabalawI \\ Department of Electrical Engineering, Faculty of \\ Engineering \& Technology, University of Jordan, \\ Amman, Jordan
}

\begin{abstract}
In this paper a new method has been developed for estimating the power in maximum entropy spectral analysis. The method is based on estimating the power in the sequence domain, where the residues associated with the poles of the prediction-error filter have been used in the estimation process.

The proposed method utilizes Parseval's theorem and it eliminates to a great extent the cross-correlation between the components of the signal and the corrupting noise.

A number of examples have been studied and a sample is presented to demonstrate the potentiality of the proposed estimation process. The results obtained indicate that the performance of this power estimation technique is superior to the estimation process which is based on the residues.
\end{abstract}

\section{Introduction}

Spectral estimation is the problem of determining the distribution in the frequency domain of the power of a random process. This problem has received a special attention due to its importance. A number of technical papers related to spectral estimation are available in the literature ${ }^{[1-5]}$.

The power spectral density associated with a random process $x(n)$ can be estimated by modeling the process $x(n)$ as the output of a parametric system excited by white noise as shown in Fig. 1. 


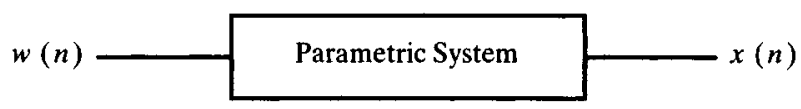

FIG. 1.

The parametric system can be described in the $z$-domain by a polynomial $A_{p}(z)$ of order $P$ such that

$$
A_{p}(z)=\sum_{i=0}^{p} a_{i} z^{-i}
$$

where $a_{i}$ are the coefficients of the parametric system and are often called model parameters.

The power spectral density $S_{x}(f)$ of the finite sequence $x(n)$ can be estimated from the model parameters. Often, autoregressive (AR) techniques are used for determining the model parameters. The length of model parameters in general is infinite since the modeling is based on an infinite number of past samples. However when the process $x(n)$ is AR, it can be modeled by an all-pole filter.

Three techniques are widely used for estimating the model parameters from a given observed segment of data. Those techniques are the Yule-Walker method ${ }^{[6]}$, the covariance method ${ }^{[7]}$, and the Burg's (maximum entropy) method ${ }^{[8]}$.

In this paper the maximum entropy method (MEM) will be used for estimating the power spectral density, because of its high resolution characteristics.

The ME power spectral estimator is given by

$$
S_{x}(f)=\frac{E_{p} t_{s}}{\left|1-\sum_{i=1}^{p} a_{i} e^{-j 2 \pi f t_{s}}\right|^{2}}
$$

where the frequency $f$ is limited to the Nyquist interval, i.e.

$$
-\frac{1}{2 t_{s}} \leq f \leq \frac{1}{2 t_{s}}
$$

$E_{p}$ is the output of a $(P+1)$ long prediction error filter with coefficients $\left(1,-a_{1}, \cdots,-a_{p}\right)$. $E_{p}$ and the model coefficients are related through the normal equations

$$
\left(\begin{array}{cccc}
R_{0} & R_{1} & \cdots & R_{p} \\
R_{1} & R_{0} & \cdots & \cdot \\
\cdot & \cdot & & \cdot \\
\cdot & \cdot & & \cdot \\
\cdot & \cdot & & \cdot \\
R_{p} & \cdot & \cdots & R_{0}
\end{array}\right)\left(\begin{array}{c}
1 \\
-a_{1} \\
\cdot \\
\cdot \\
\cdot \\
-a_{p}
\end{array}\right)=\left(\begin{array}{c}
E_{p} \\
0 \\
\cdot \\
\cdot \\
\cdot \\
\cdot
\end{array}\right)
$$


The elements of the $R$ matrix are the autocorrelation with lag $P$. This matrix is a toeplitz one and an efficient algorithm for calculating those coefficients through the utilization of Levenson's recursion is found in reference [9].

Having estimated the power spectral density, another spectral feature that is of importance and needs accurate estimation, is the power content in a given signal around a certain frequency band. In reference [10], a conceptually simple method for power estimation in maximum entropy spectral analysis was presented. This method is based on the evaluation of the complex residues of the power spectral density estimator. This method was found to be efficient and particularly suitable for spectral decomposition in low noise environments. However when noise power starts to increase and the SNR approaches $0 \mathrm{~dB}$ and below, the method starts to poorly perform. This may be attributed to the fact that the estimated power contains the noise power as an inherent part of it. In this paper the same problem will be addressed but by using a different approach, and the resulting power estimation is almost noise free.

The rest of this paper is organized as follows. In section 2 , the problem under investigation is formulated. The newly developed approach for power estimation is presented in section 3 . In section 4 , the performance of the proposed estimation technique is evaluated by using computer simulation. In addition, the results obtained are compared with the previous method. Some concluding remarks are given in section 5 .

\section{Problem Formulation}

Consider the random process $x(t)$. This process can be modeled by a set of $A R$ parameters as follows

$x(t)=-a_{1} x\left(t-t_{s}\right)-a_{2} x\left(t-2 t_{s}\right)-\cdots-a_{p} x(t-p t s)+w(t)$

The problem under consideration can be stated as follows, given the set of $A R$ parameters, then it is required to estimate the power content of the signal.

The signal $x(t)$ can be looked at as a sum of sinusoids corrupted with noise. Therefore (5) can be rewritten in the form

$$
\begin{aligned}
x(t) & =s(t)+w(t) \\
& =\Sigma A_{i} \sin \left(\omega_{i} t+\Theta_{i}\right)+w(t)
\end{aligned}
$$

The signal $x(t)$ has been observed (sampled) at instants of time separated by $t_{s}$. The output of the sampling process will be a vector of data points. This vector is called the sample vector and is given by

$$
x=\left[x_{0}, x_{1}, \cdots, x_{\mathrm{N}-1}\right]
$$

where $N$ samples are considered.

The noise samples are independent with zero mean, normally distributed with var- 
iance $\sigma^{2}$.

Without loss of generality, we may assume that $t_{s}=1$. On the unit circle in the $z$ plane, eq. (2) can be rewritten in the form

$$
S_{x}(f)=\left.\frac{E_{p}}{A_{p}(z) A_{p}\left(z^{-1}\right)}\right|_{z}=e^{j \pi f}
$$

The roots of Schur-Cohn polynomial $A_{p}(z), z_{1}, z_{2}, \cdots, z_{p}$ lie inside the unit circle in the $z$-plane ${ }^{[11]}$. Consequently the roots of $A_{p}\left(z^{-1}\right)$ lie outside that circle. The aforementioned constraint on the roots of $A_{p}(z)$ insures the stability of the AR model.

To estimate the power content we may use the frequency domain approach. This approach is based on residue integral theory. The power associated with the sequence $x(n)$ is given by

$$
P_{0}=\sum_{-\infty}^{\infty} x(n) x^{*}(n)
$$

by using the $z$-transform we obtain

$$
P_{0}=\sum_{-\infty}^{\infty} x(n) x^{*}(n)=\frac{1}{2 \pi j} \int_{c} X(z) X^{*}\left(z^{-1}\right) \frac{d z}{z}
$$

$C$ is a Jordan contour encircling the origin. The integration path is the unit circle in the $z$-plane. $X(z)$ is the $z$-transform of $x(n)$ and is defined in terms of $E_{p}$ and the AR parameters as follows

$$
X(z)=\frac{\sqrt{E_{p}}}{A_{p}(z)}
$$

The RHS of (10) has been evaluated in reference [10] by using the residue integral theory. The estimate of the total power $P_{0}$ in $x(t)$ has been formulated as

$$
P_{0}=\sum_{k=1}^{p} \operatorname{Res}\left(z_{k}\right)
$$

where $\operatorname{Res}\left(z_{k}\right)$ is the residue of $S_{x}(z) / z$ at $z=z_{k}$ and is given by

$$
\operatorname{Res}\left(z_{k}\right)=\frac{E_{p}}{\left(1-z_{k} z_{k}^{*}\right) \prod_{j \neq k}\left(z_{k}-z_{j}\right)\left(z_{k}^{-1}-z_{j}^{*}\right)}
$$

Also in reference [10], it has been found that the power at the resonance $f=f_{k}$ is given by

$$
\begin{aligned}
P_{0}\left(f_{k}\right) & =2 \operatorname{Res}\left[\operatorname{Res}\left(z_{k}\right)\right], 0<f_{k}<\frac{1}{2} \\
& =\operatorname{Res}\left[z_{k}\right], \quad f_{k}=0 \text { or } \frac{1}{2}
\end{aligned}
$$


This estimate will be called the residue estimate.

\section{Sequency-Domain Approach for Power Estimation}

In the previous section, the frequency domain has been used to estimate the power in the process $x(t)$. In this section a new approach will be developed.

The power can be estimated by evaluating the summation in the LHS of (10), i.e., by using (9). To perform this operation, initially we have to evaluate the sequence $x(n)$ by using the inverse $z$-transform of $X(z)$ which is defined in (11).

$$
\begin{aligned}
x(n) & =Z^{-1}[X(z)] \\
& =\sqrt{E_{p}} Z^{-1}\left[\frac{1}{A_{p}(z)}\right]
\end{aligned}
$$

The all-pole function $X(z)$ can be rewritten in the summation form as follows

$$
X(z)=\sum_{j=1}^{p} X_{j}(z)
$$

where

$$
X_{j}(z)=\frac{\sqrt{E_{p}} R_{j}}{\left(1-z^{-1} z_{j}\right)}
$$

$R_{j}$ is the residue of $X(z)$ at $z=z_{j}$ and is given by

$$
R_{j}=\frac{1}{\prod_{j=1, j \neq k}^{p}\left(1-z_{k} z_{j}^{-1}\right)}
$$

since all the zeros of $A_{p}(z)$ lie inside the unit circle, then the ROC (region of convergence) of the inverse- $z$ transform of $x_{j}(t)$ is $|z|>1$. Therefore $x_{j}(n)$ can be expressed as

$$
x_{j}(n)=\sqrt{E_{p}} R_{j} z_{j}^{-n} u(n)
$$

Each term in (16) can be considered as a signal resonating at a frequency that can be estimated from the argument of the root corresponding to it as illustrated in reference [12].

$$
f_{k}=\frac{\operatorname{Im}\left[\ln \left(z_{k}\right)\right]}{2 \pi t_{s}}
$$

and the bandwidth estimated by

$$
B w_{k}=\frac{\ln \left|z_{k}\right|}{\pi t_{s}}
$$

The roots may be either real or complex, therefore the signals $X_{j}(z), j=1, \cdots, p$ will 
be either real or complex. However, when they are complex, they appear in complex conjugate pairs. Therefore, by using this terminology, the overall sequence can be considered as the summation of the individual terms, and this will be real.

The signal $x(n)$ can be decomposed into its contributing parts $x_{j}(n)$ such that each subsequence is concentrated around a certain resonance frequency $f_{j}$.

The total power found in the neighborhood of the frequency $f_{j}$ can be estimated as the zero lag autocorrelation of $x_{j}(n)$ (auto-power) plus the cross-correlation of $x_{j}(n)$ with $x_{i}(n), i=1,2, \cdots, j-1, j+1, \cdots, p$ (cross-power). The above concept yields

$$
P_{0}\left(f_{j}\right)=\sum_{-\infty}^{\infty} x_{j}(n) x_{j}^{*}(n)+\sum_{-\infty}^{\infty} x_{j}(n) \sum_{\substack{i=1 \\ i \neq j}}^{p} x_{i}^{*}(n)
$$

The substitution of (18) in (20) produces

$$
\begin{aligned}
P_{0}\left(f_{j}\right) & =E_{p}\left[\sum_{0}^{\infty}\left|R_{j}\right|^{2}\left|z_{j}\right|^{2 n}+\sum_{\substack{i=1 \\
i \neq j}}^{p} \sum_{n=0}^{\infty} R_{j} R_{i}^{*}\left(z_{j} z_{i}^{*}\right)^{n}\right] \\
& =E_{p} \sum_{i=1}^{p} \frac{R_{j} R_{i}^{*}}{1-z_{j} z_{i}^{*}}
\end{aligned}
$$

Now if the sequence $x_{j}(n)$ is complex, then its complex conjugate $x_{j}^{*}(n)$ will contribute to the power in the same frequency band. Therefore the total power around $f_{j}$ will be the summation of the power in the signals $x_{j}(n)$ and $x_{j}^{*}(n)$

$$
\begin{aligned}
P_{0}\left(f_{j}\right) & =P_{x_{j}}\left(f_{j}\right)+P_{x_{j}}^{*}\left(f_{j}\right) \\
& =2 E_{p} \operatorname{Re}\left[\sum_{i=1}^{p} \frac{R_{j} R_{i}^{*}}{1-z_{j} z_{i}^{*}}\right]
\end{aligned}
$$

By virtue of Parseval's theorem, this power estimate is exactly the same as the power estimate found by using the frequency domain approach.

The total power in the signal $x(n)$ is the summations of the powers in all $x_{j}(n)$, i.e.

$$
P_{0}=E_{p} \sum_{j=1}^{p} \sum_{i=1}^{p} \frac{R_{j} R_{i}^{*}}{1-z_{j} z_{i}^{*}}
$$

It is evident from the past development that, both the residue power estimate, and the power estimate of (23) contain as part of them the cross power between the signals $x_{j}(n)$ and the remaining components. This fact is not clear in the residue estimate method formulation. However one may think of another estimate for the power that depends on the auto-power resulting from the correlation of the signal 
$x_{j}(n)$ with itself only. This estimate is given by

$$
P_{0}\left(f_{j}\right)=\frac{2 E_{p} R_{j} R_{j}^{*}}{1-z_{j} z_{j}^{*}}
$$

The factor of 2 has appeared in (24) to account for the power from the conjugate $x_{i}^{*}(n)$. It is expected that the estimate of $(24)$ will give more accuratein the signal $x(n)$ at $f_{j}$ than the residue estimate.

\section{Performance Evaluation}

The concepts presented in the previous section can be illustrated by the following examples. The first example is the same as that found in reference [10], where the residue estimate was derived.

\section{Example 1}

Consider the following autocorrelation function, $R_{n}$ for $n=0, \cdots, 10$

$$
R_{n}=\delta_{0 n}+p_{1} \cos \left(2 \pi n / \lambda_{1}\right)+p_{2} \cos \left(2 \pi n / \lambda_{2}\right)
$$

where $\delta_{0 n}$ is the Kronecker deltá. The powers $p_{1}$ and $p_{2}$ are then varied, keeping the white noise power fixed at unity. The $A R$ model parameters were calculated from the $11 \times 11 R$ matrix using Levenson's algorithm. A computer program in Fortran has been written to calculate the parameters of the AR model and to simulate equation $(24)^{[13]}$. Column 5 in Table 1 lists the corrected power estimates, where the correction mechanism is indicated at the end of this example.

Table 1 lists the estimated wavelengths, bandwidths, and the corresponding power estimates using the two methods. Column 1 shows the estimated wavelengths, column 2 lists the estimated bandwidths, column 3 lists the residue estimates and column 4 lists the power estimates based on (24).

As expected the estimates of (24) are closer to the power of the sinusoids than the residue estimate. This difference is due to the cross-correlation between the various

TABle 1. Powers, wavelengths, and bandwidths obtained from estimates based on $R_{n}$ of Example 1. This

\begin{tabular}{|c|c|c|c|c|}
\hline Wavelength & Bandwidth & Res. est. & Est. (24) & Corr. est. \\
\hline 4.997 & $7.80 \mathrm{E}-004$ & 10.88 & 10.67 & 10.68 \\
\hline 2.472 & $5.26 \mathrm{E}-002$ & $1.91 \mathrm{E}-001$ & $6.03 \mathrm{E}-002$ & - \\
\hline 10.006 & $1.65 \mathrm{E}-003$ & 5.55 & 5.33. & 5.35 \\
\hline 3.239 & $4.39 E-002$ & $1.93 \mathrm{E}-001$ & $6.82 \mathrm{E}-002$ & - \\
\hline 0.000 & 0.00 & $8.67 \mathrm{E}-002$ & $2.43 \mathrm{E}-002$ & _- \\
\hline 0.000 & 0.00 & $9.54 \mathrm{E}-004$ & $2.93 \mathrm{E}-002$ & - \\
\hline Total power & & 16.99 & 16.19 & \\
\hline
\end{tabular}
table should be compared with Table 1 of reference [10]. 
Table 1. Contd.

(b) : $p_{1}=2.66, p_{2}=5.33$

\begin{tabular}{|c|l|l|l|c|}
\hline Wavelength & Bandwidth & Res. est. & Est. (24) & Corr. est. \\
\hline & & & & 2.66 \\
\\
10.012 & $3.23 \mathrm{E}-003$ & 2.88 & 5.35 & 5.35 \\
2.995 & $1.55 \mathrm{E}-003$ & 5.55 & $5.98 \mathrm{E}-002$ & - \\
3.239 & $5.32 \mathrm{E}-002$ & $1.91 \mathrm{E}-001$ & $6.76 \mathrm{E}-002$ & - \\
0.000 & $4.45 \mathrm{E}-002$ & $1.93 \mathrm{E}-001$ & $2.42 \mathrm{E}-002$ & - \\
0.000 & 0.00 & $8.68 \mathrm{E}-002$ & $2.90 \mathrm{E}-002$ & - \\
\hline Total power & 0.00 & $9.54 \mathrm{E}-002$ & 8.19 & \\
\hline
\end{tabular}

(c) : $p_{1}=1.33, p_{2}=2.66$

\begin{tabular}{|c|c|c|c|c|}
\hline $\begin{array}{r}4.99 \\
2.47 \\
10.02 \\
3.24 \\
0.00\end{array}$ & $\begin{array}{l}3.08 E-003 \\
5.45 E-002 \\
6.17 E-003 \\
4.57 E-002 \\
0.00\end{array}$ & $\begin{array}{l}2.88 \\
1.91 \mathrm{E}-001 \\
1.55 \\
1.93 \mathrm{E}-001 \\
8.71 \mathrm{E}-002\end{array}$ & $\begin{array}{l}2.68 \\
5.87 \mathrm{E}-002 \\
1.34 \\
6.63 \mathrm{E}-002 \\
2.34 \mathrm{E}-002\end{array}$ & $\begin{array}{c}2.68 \\
- \\
1.35 \\
- \\
-\end{array}$ \\
\hline Total power & & 2.99 & 2.19 & \\
\hline \multicolumn{5}{|c|}{ (d) : $p_{1}=0.66, p_{2}=1.33$} \\
\hline $\begin{array}{r}4.98 \\
2.47 \\
10.05 \\
3.24 \\
0.00 \\
0.00\end{array}$ & $\begin{array}{l}6.00 E-003 \\
5.69 E-002 \\
1.14 E-002 \\
4.80 E-002 \\
0.00 \\
0.00\end{array}$ & $\begin{array}{l}1.55 \\
1.91 E-002 \\
8.76 E-001 \\
1.93 E-001 \\
8.71 E-002 \\
9.53 E-002\end{array}$ & $\begin{array}{l}1.35 \\
5.69 \mathrm{E}-002 \\
6.71 \mathrm{E}-001 \\
6.41 \mathrm{E}-002 \\
2.34 \mathrm{E}-002 \\
2.77 \mathrm{E}-002\end{array}$ & $\begin{array}{l}1.35 \\
- \\
6.76 \mathrm{E}-001 \\
- \\
- \\
-\end{array}$ \\
\hline Total power & & 2.99 & 2.19 & \\
\hline \multicolumn{5}{|c|}{ (e) : $p_{1}=0.33, p_{2}=0.66$} \\
\hline $\begin{array}{r}4.97 \\
2.47 \\
10.08 \\
3.24 \\
0.00 \\
0.00\end{array}$ & $\begin{array}{l}1.15 E-002 \\
6.10 E-002 \\
1.97 E-002 \\
5.19 E-002 \\
0.00 \\
0.00\end{array}$ & $\begin{array}{l}0.80 E-001 \\
1.91 E-001 \\
5.44 E-001 \\
1.93 E-001 \\
8.74 E-002 \\
9.52 E-002\end{array}$ & $\begin{array}{l}6.83 E-001 \\
5.41 E-002 \\
3.48 E-001 \\
6.06 E-2 \\
2.26 E-002 \\
2.63 E-002\end{array}$ & $\begin{array}{l}6.80 \mathrm{E}-001 \\
- \\
3.44 \mathrm{E}-001 \\
- \\
- \\
-\end{array}$ \\
\hline Total power & & 1.99 & 1.19 & \\
\hline
\end{tabular}

resonances that is found in the residue estimate but not in the estimate of (24). Certainly the cross powers are not as a result of cross-correlation between the sinusoids, but they are in fact due to cross-power between the white noise components present 
at the corresponding frequencies.

The total estimated power using the residue estimate is equal, in all cases, to the total power in the signal $x(n)$. This means that noise power is affecting the residue estimate and to eliminate this effect, the amount of noise power present around a certain frequency will be subtracted from the residue estimate corresponding to that frequency. The model order here is 10 , thus the AR model can represent a system with five resonances. These resonances tend to share equally the power of the white noise thus, a power of 0.2 was subtracted from the powers of column 2 , and the result is tabulated in column 5 .

It is obvious that the results in columns 4 and 5 are approximately the same. This confirms the fact that the estimate of $(24)$ is free from cross power of white noise. To illustrate this point further another example will be given.

\section{Example 2}

Consider the same autocorrelation function of example 1 but this time the powers $p_{1}$ and $p_{2}$ will be fixed at 2.66 and 1.33 respectively while changing the added noise power from 1.51 to 1000 . The results are listed in Table 2 , and are further illustrated in Fig. 2.

TABLE 2. Powers obtained using the residue estimate and estimate of (24) for the autocorrelation function given in Example 2.

\begin{tabular}{|l|r|r|r|r|r|r|}
\hline Added noise power & 1.51 & 6.01 & 16.01 & 46.01 & 96.01 & 146.01 \\
\hline & $p_{1} 1.66$ & 2.61 & 4.68 & 10.84 & 21.06 & 31.26 \\
Residue estimate & $p_{1} 1.35$ & 1.41 & 1.48 & 1.64 & 1.86 & 2.06 \\
Corr. res. est. & $p_{1} 1.34$ & 1.47 & 1.84 & 2.99 & 4.79 & 6.48 \\
Est. of (24) & $p_{2} 2.99$ & 3.99 & 6.25 & 12.95 & 23.96 & 34.84 \\
Residue estimate & $p_{2} 2.69$ & 2.79 & 3.04 & 3.75 & 4.76 & 5.65 \\
Corr. res. est. & $p_{2} 2.69$ & 2.81 & 3.20 & 4.46 & 6.47 & 8.36 \\
Est. of (24) & & & & & & \\
\hline
\end{tabular}

It is seen that as the added noise power increases, the error between the corrected estimate and the estimate of (24) increases. It is noteworthy that the corrected residue estimate curve branches off the curve of the present estimate for noise power larger than 20. For higher noise power the estimate of (24) yielded higher estimates than the corrected residue estimate. The explanation to this observation lies in the fact that we are dealing here with truncated sinusoids. It is well known from the basic theory of communications, that under such conditions the spectrum of the sinusoid will no longer be a delta function but rather more or less like a sampling function. This function is characterized by the presence of sidelobes in its response. Therefore, the higher estimates of (24) can be referred to the sidelobes resulting from truncating the sinusoids.

To illustrate this point further another example will be presented where only one sinusoid is present to preclude the effect of interference. 


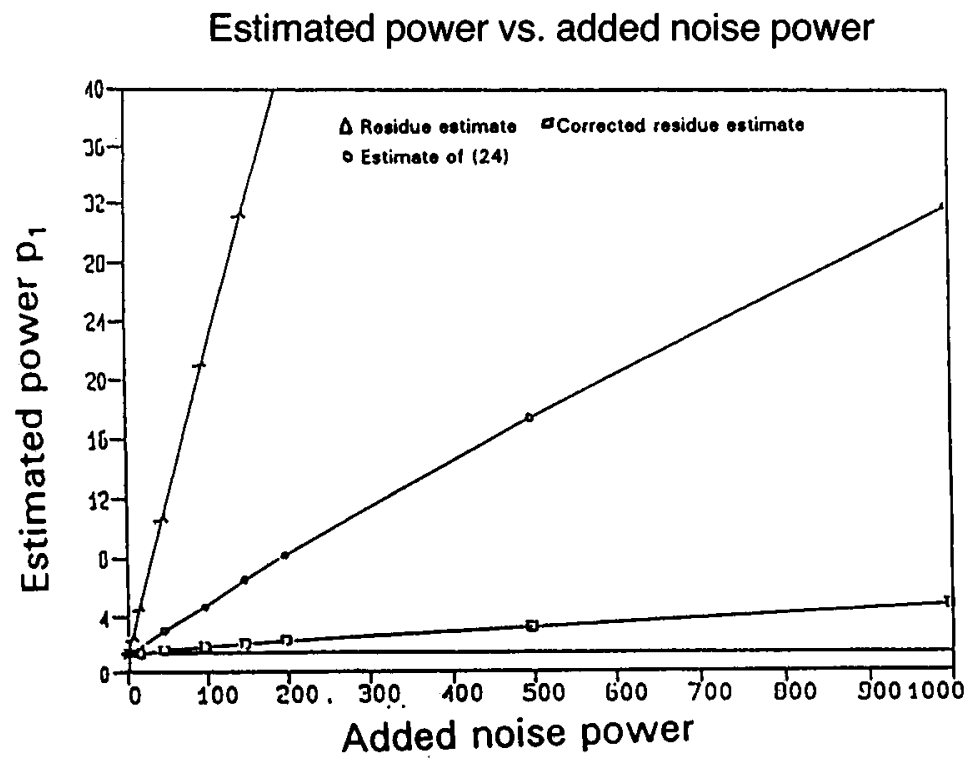

(a)

Estimated power vs. added noise power

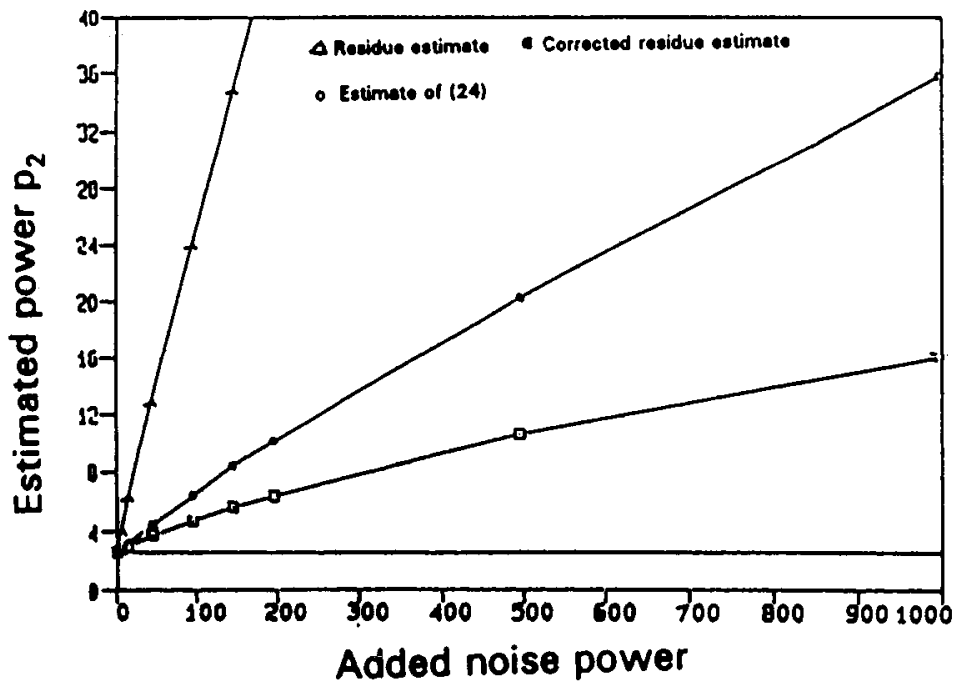

(b)

FIG. 2. Variation of the estimated powers with added noise power (Two sinewaves) (a) $p_{1}$, (b) $p_{2}$. 


\section{Example 3}

Consider the same autocorrelation function of the previous example after eliminating the sinusoid with $p=1.33$. The powers are then estimated in the same way and the results are listed in Table 3 and shown in Fig. 3.

TABlE 3. Powers estimated using the residue method and from (24) for the autocorrelation given in Example 3.

\begin{tabular}{|l|c|c|c|c|r|r|}
\hline Added noise power & 1 & 2.84 & 7.34 & 17.34 & 47.34 & 97.34 \\
\hline & & & & & & \\
Residue estimate & 2.9 & 3.53 & 4.45 & 6.85 & 13.90 & 25.36 \\
Corr. res. est. & 2.7 & 2.96 & 2.98 & 3.38 & 4.44 & 5.89 \\
Est. of (24) & 2.69 & 2.76 & 2.95 & 3.49 & 4.73 & 6.79 \\
\hline
\end{tabular}

\section{Estimated power vs. added noise power}

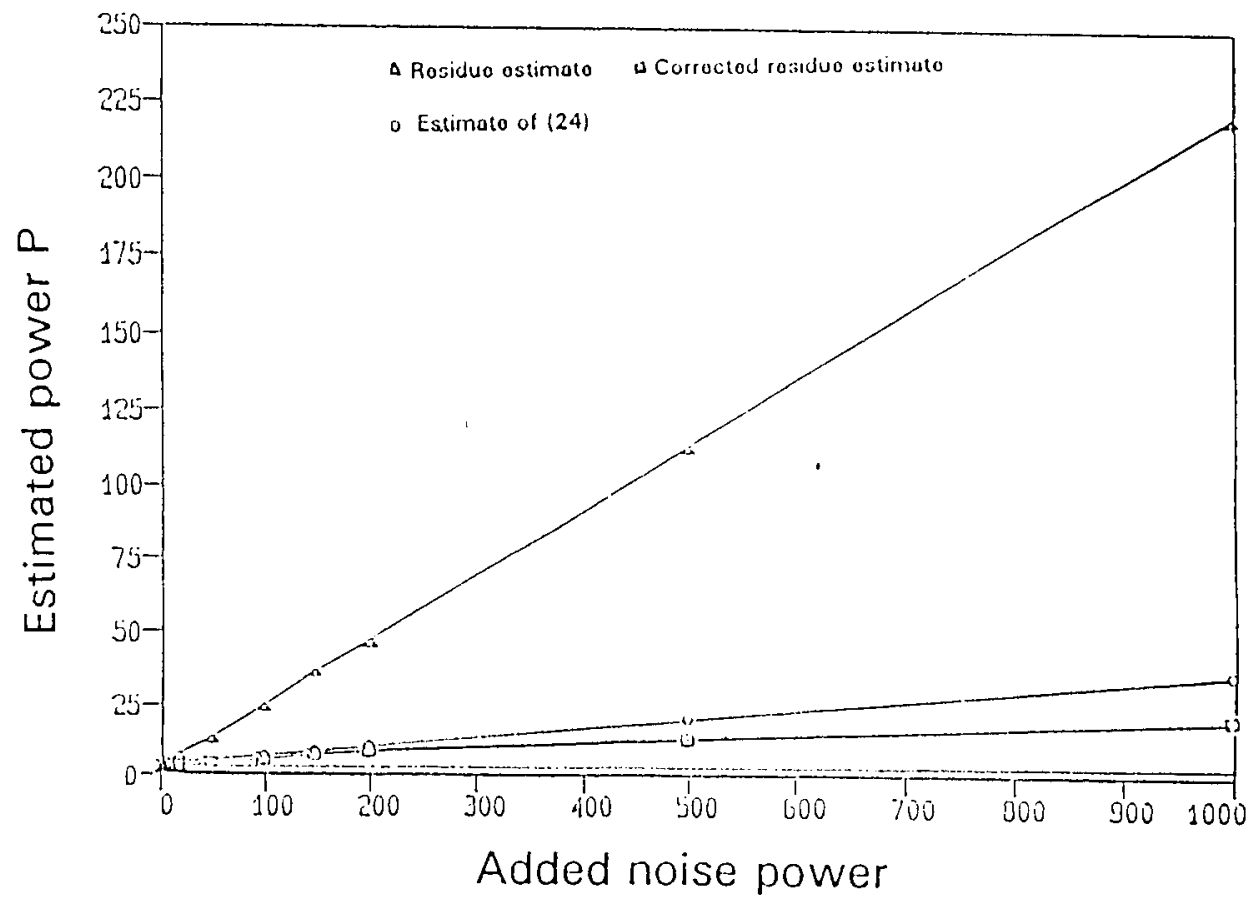

FIG. 3. Variation of estimated power with added noise power (single sinewave).

It is obvious from Table 3 and Fig. 3 that no matter how large is the value of the added noise power, the estimate of $(24)$ and the corrected residue estimates are approximately the same. This result confirms the aforementioned argument.

Therefore, it can be concluded that the estimate of (24) is very efficient in retrieving signals buried in noise without incorporating any prior knowledge. 
Another important aspect concerning power estimation that still requires further investigation is the study of the behavior of the two estimates for the case of two close lying harmonic components. The next example serves to illustrate this effect clearly.

\section{Example 4}

Consider the following autocorrelation $R_{n}$ for $n=0,1$

$$
R_{n}=\delta_{0 n}+\cos \left(2 \pi n / \lambda_{1}\right)+\cos \left(2 \pi n / \lambda_{2}\right)
$$

corresponding to two sine waves of equal power and rather close wave lengths $\lambda_{1}=$ $20, \lambda_{2}=18$. The AR model order $P$ was varied from 10 to 40 and the corresponding powers are estimated in the same way as the previous example. Figure 4 shows the variation of the estimated powers with $P$.

\section{Estimated power vs. model order $P$}

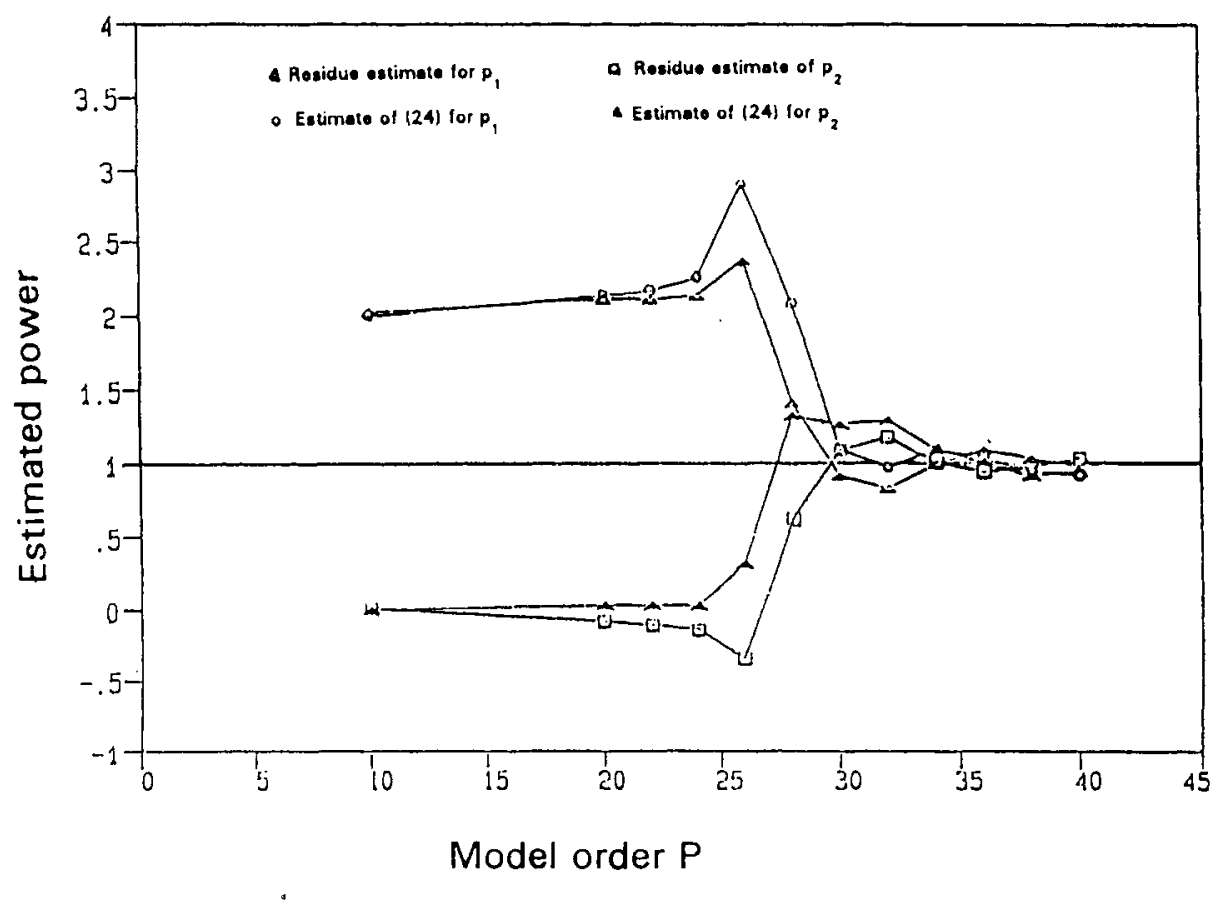

Fig. 4. Variation of estimated power with the model order $P$.

One can easily note here the transient occurrence of a negative power for the residue estimate, which can be easily explained by the presence of a negative crosspower, (resulting from correlation between the corresponding pole with all the remaining ones) that is larger than the auto-power resulting from the correlation of the corresponding pole with itself. This fact, however, is not directly evident from the frequency domain approach expression. 
Figure 4 also shows the curves for the true values of $p_{1}$ and $p_{2}$. When comparing the two estimates it is noted that these estimates become equal for large order values. This is because as $P$ increases the contribution from the added noise power becomes smaller and smaller and in the end the two estimates become equal. It can be also seen that the estimate of (24) is never negative since it results from the product of two complex conjugate numbers. This fact makes the estimate of (24) physically more convenient.

\section{Discussion}

The results presented in section 4 indicate that the new power estimation method is useful to extract signals embedded in background white noise. Further, it has been shown that the time domain approach for the derivation of the corresponding power estimate is to be preferred over the older frequency domain approach. This is because the former gave more insight into the various components (auto- and crosscorrelation), participating in the overall power estimate, and thus the explanation of the negative power calculated under certain conditions is straight forward.

\section{List of Symbols}

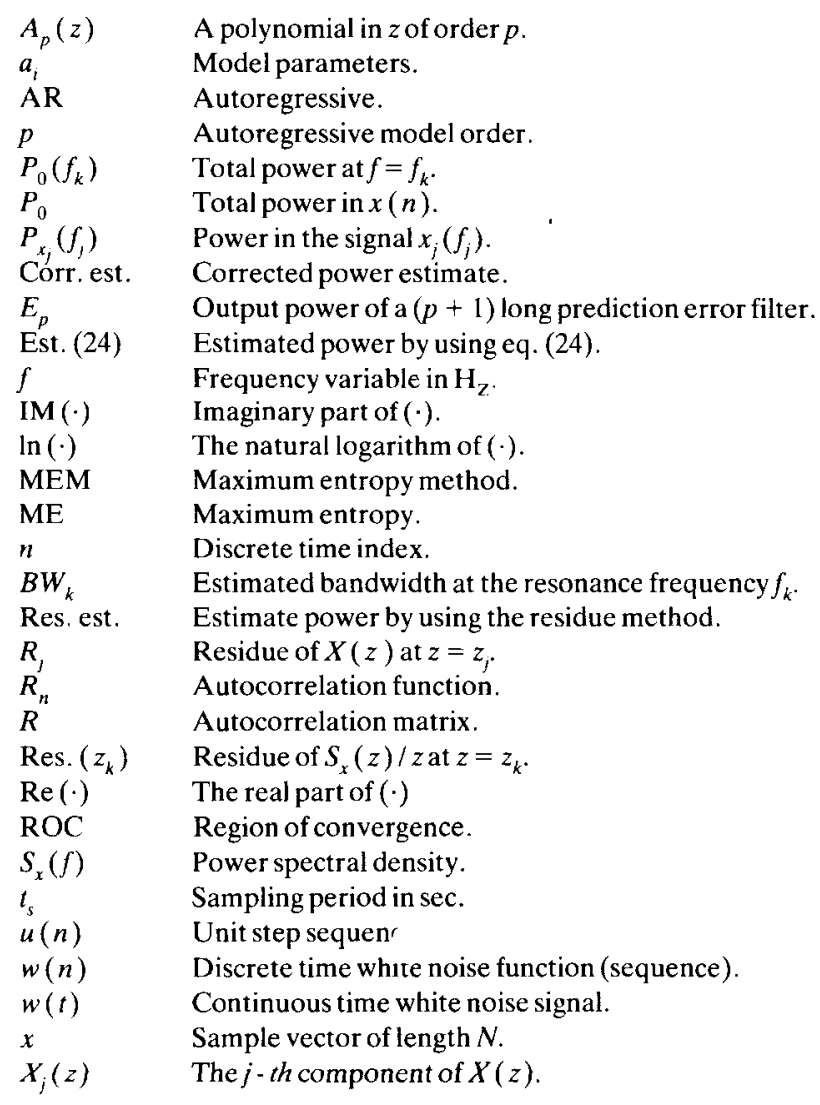




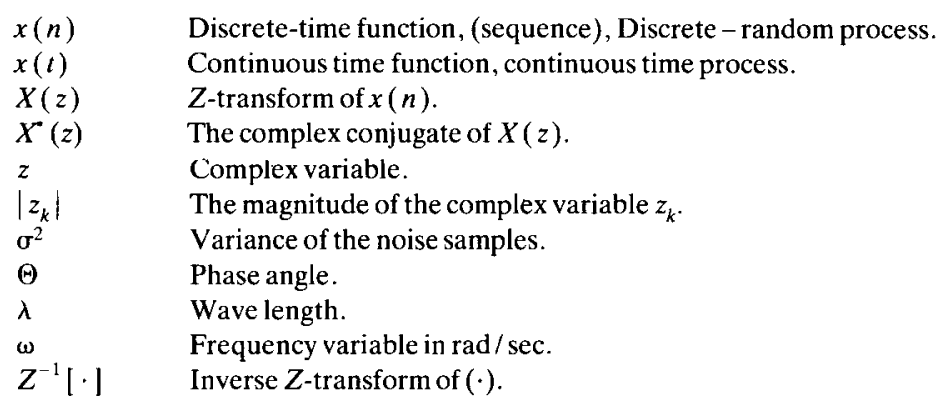

\section{References}

[1] Kay, S., Modern Spectral Estimation: Theory and Application, Prentice-Hall, Englewood Cliffs, NJ, pp. 217-252 (1987).

[2] Kay, S. and Marple, S.L., Jr., Spectrum analysis: A modern perspective, Proc. IEEE, 69 (Nov.): 1380-1419 (1981).

[3] Marple, S.L., Jr. A new autoregressive spectrum analysis algorithm, IEEE Trans. Acoustics, Speech and Signal Processing, ASSP-28 (Aug.): 441-454 (1980).

[4] Makhoul, J., Linear prediction: A tutorial review, Proc. IEEE, 63: 561-580 (1975).

[5] .Haykin, S., (Ed.), Nonlinear Methods of Spectral Analysis, Springer-Verlag, New York, pp. 155-179 (1979).

[6] Markel, J.D. and Gray, A.H., Jr., Linear Prediction of Speech, NY: Springer-Verlag, New York, Vol. 12 (1976).

[7] Atal, B.S. and Hanauer, S., Speech analysis and synthesis by linear prediction of speech wave, $J$. Acoust. Soc. Amer., 50(2): 637-670 (1971).

[8] Burg, J.P., Maximum Entropy Spectral Analysis, 37th Annual Int. SEG Meeting, Oklahoma City (1967).

[9] Orfanidis, S.J., Optimum Signal Processing: An Introduction, NY: Macmillan Publishing Company, New York, pp. 81-85 (1985).

[10 Johnson, S.J. and Anderson, N., On power estimation in maximum entropy spectral analysis, Geophysics, 43(4) June: 681-690 (1978).

[11] Haykin, S., Modern Filter, Macmillan Publishing Company, New York, pp. 108-152 (1989).

[12] Schoeder, M.R., Linear Prediction Entropy and Signal Analysis, IEEE ASSP Magez., 1 (July): 3-11 (1984).

[13] Kalouptisdis, N. and Theodoridis, S., Fast Adaptive Least Squares Algorithm for Power Spectral Estimations, IEEE Trans. ASCP, 35(5) May: 661-670 (1987). 


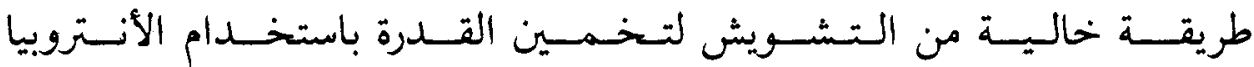

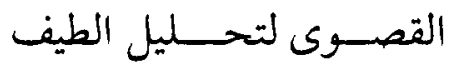

\section{مهاب دقـه و عصام زعبلاوي}

قسم الهندسة الكهربائية ، كلية الهندسة والتكنولوجيا ، البحامعة الأردنية

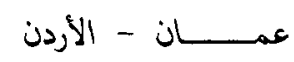

المستخلصى . يتنـاول هذا البحث طريقة جديلة لتخمـين القلدرة المتضمنة في متتابعة

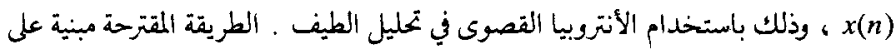
حساب القدرة باستخدام نظرية بارسيفال وهي تحذف إلى حل بعيد الترابط المتبادل بين

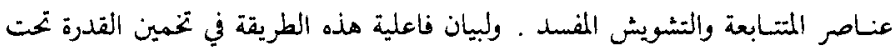

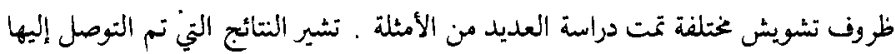

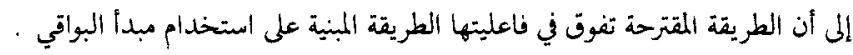

